\title{
A Boolean Algebra Based Learnable Network
}

\author{
Non-member Zheng Tang \\ (Miyazaki University) \\ Non-member Makoto Kuratsu (Miyazaki University) \\ Non-member Okihiko Ishizuka (Miyazaki University) \\ Non-member Koichi Tanno (Miyazaki University)
}

We present a learnable Boolean algebra based network. The learnable network is derived directly from Boolean algebra, e.g., a canonic sum-of-minterms realization of a logic function. However, the conventional back-propagation algorithm cannot be applied to the logical networks whose processing units have logic AND, logic OR operators because the required derivatives are not available. In this paper, we use a pseudo-neuron, a softmin and a softmax functions to develop an algorithm similar to back-propagation, but for the logical AND-OR based networks. It is shown that the networks are capable of learning just like neural networks, and the computational framework of the algorithm is also similar to standard back-propagation, but its hardware implementations are unrivaled in simplicity and speed when applied to a binary-to-binary mapping. Several examples are given which demonstrate its applicability and simulate some biological cytology properties successfully.

Keywords: logic network, Boolean algebra, learning, back-propagation

\section{Introduction}

In recent years, the neural networks and their backpropagation training algorithm ${ }^{(1)}$ have gained considerable attention. These technologies have been applied successfully to many engineering disciplines ${ }^{(2) \sim(4)}$. Neural network can supplement the enormous processing power of the von-Neumann digital computer with the ability to make sensible decisions and to learn by ordinary experience, as we do. However, the hardware implementation of the neural networks and the backpropagation algorithm come into question. Digital designs, such as systolic arrays ${ }^{(5)}$ allow straightforward application of the learning algorithm and easy adaptation to different network topology, but they suffer from the latency associated with multiplexing computation operations. The time required to compute the effect of a single connection is significantly greater than that required by the corresponding operation in an analogue neural network. However, existing analogue circuits are also not without exception. Most of them employ operational amplifiers and resistive connection element ${ }^{(6)(7)}$ to provide one effective medium for implementing natural neuron functions. They have physical limitations in terms of response speed, circuit area and power dissipation. They also have functional limitations involving synapse programming ability and the implementation of on-chip adaptation ${ }^{(8)(9)}$.

Meanwhile digital systems such as the modern vonNeumann digital computers, have almost attained perfection in high-speed and high-density. But, the backpropagation algorithm cannot be applied directly to the digital networks of units with logical AND, OR func- tions, and there is no theoretical justification for a gradient descent technique since these functions cannot be differentiated. However, if the logical AND, OR function are replaced by a softmin function, and a softmax function, they are both continuously differentiable functions. Furthermore, by adding a pseudo-neuron between the input and AND cells, we can represent the connection states and use gradient descent to optimize the weighs and thresholds of the pseudo-neuron, so that the connection states ${ }^{(10)}$.

The advantages of the schemes are that the hardware implementations of the learnable network are dramatically less costly than that of the conventional neural networks, and their speed is also vastly increased over the conventional neural networks. This is due to the fact that the learnable networks do not use any analog or digital neuron cells, but only logic AND, logic OR gates. The learning in the architecture is considerably simple, since it involves only logic operations when applied to digital systems.

The paper is organized as follows. In Section 2 we describe the general architecture (e.g., the AND-OR architecture) of the network while in Section 3 we introduce the pseudo-neuron, the softmin and softmax functions to represent the connection states, the logic AND operators and the logic OR operators. Section 4 provides details concerning the learning algorithm while Section 5 presents simulations and describes some biological cytology properties, such as the cell adhesion, the death of cell, and the death of cell cluster, shared by the learnable networks. The paper is concluded with some remarks in Section 6. 


\section{Logic Networks}

To facilitate our analysis and synthesis of the learnable networks, let us recall a few fundamental switching algebra. If we can get simple switch expressions by algebraic manipulation, we can obtain simple networks. The truth table, the Karnaugh map and the algebraic expression are conveniently used in logic design. These will be subsequently used as aids to help explain the operation of the learnable networks.

Switching algebra is an algebraic system that consists of the set 0,1 (i.e., we deal with only two consists, 0 and 1) and operations of AND, OR and NOT. Thus, any logic function can be uniquely expanded with a canonical sum-the AND-OR network shown. For example, a logic function as shown in Table 1 can be written as

$$
\begin{aligned}
F= & \overline{x_{1}} \overline{x_{2}} x_{3} x_{4}+\overline{x_{1}} x_{2} \overline{x_{3}} \overline{x_{4}}+\overline{x_{1}} x_{2} \overline{x_{3}} x_{4} \\
& +\overline{x_{1}} x_{2} x_{3} \overline{x_{4}}+\overline{x_{1}} x_{2} x_{3} x_{4}+x_{1} \overline{x_{2}} \overline{x_{3}} \overline{x_{4}} \\
& +x_{1} \overline{x_{2}} x_{3} x_{4} \ldots \ldots \ldots \ldots \ldots \ldots \ldots \ldots \ldots
\end{aligned}
$$

where the dots "." omitted, the " + " and the overbars denote "AND", "OR" and "NOT". Alternatively, we could represent the same data in a Karnaugh map (see Table 2). We can perform logical reduction and obtain the following equation from the Karnaugh map of Table 2 :

$$
F=\overline{x_{1}} x_{2}+\overline{x_{2}} x_{3} x_{4}+x_{1} \overline{x_{2}} \overline{x_{3}} \overline{x_{4}}
$$

The logic function can be expressed as an AND-OR network as shown in Fig.1. The connection between the input layer and the AND layer has the following possible states determined by the logic function :

a) The direct connection. Input $x_{i}$ is included in logic min term $A_{N} D_{j}$. For example, since $x_{1}, x_{2}, x_{3}$ and $x_{4}$ are include in $\mathrm{AND}_{3}$ term, $\mathrm{AND}_{1}$ term and $\mathrm{AND} 2$ term, the $x_{1}, x_{2}, x_{3}$ and $x_{4}$ are connected directly to AND3, AND1 and $\mathrm{AND}_{2}$, respectively, as shown in Fig.1.

b) The inversed connection. The inverse of input

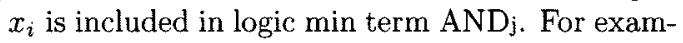
ple, since $\overline{x_{1}}, \overline{x_{2}}, \overline{x_{3}}$ and $\overline{x_{4}}$ are included to AND1 term, AND2 term and AND3 term. They are connected to AND1, AND2 and AND3 through their inverters, respectively, as shown in Fig.1.

c) 1-constant connection. Neither input $x_{i}$ nor its inverse is included in logic min term $A N D_{j}$. For example, since neither $x_{1}$ nor $\overline{x_{1}}$ is included in AND2 term, there is no connection between $x_{1}$ and AND2. That is, the input from $x_{1}$ to AND2 is always 1, i.e., 1-constant input.

d) 0-constant connection. For example, only 3 AND terms Eq.(2) are enough to realize the logic function of Table 1. Thus the four AND terms in Eq.(1) are excess. This can be accomplished by biasing a 0 -constant input to the AND gate.

Thus, with the four connection states mentioned above, any function $F$ of $n$ variables can be realized with the AND-OR network consisting of at most $2^{n-1}$ AND gates.

\begin{tabular}{|c|c|c|c|c|}
\hline $\begin{array}{r}x_{1} x_{2} \\
X_{3 X_{4}}\end{array}$ & 00 & 01 & 11 & 10 \\
\hline 00 & & 10 & & 11 \\
\hline 01 & & $\vdots 1$ & & \\
\hline 11 & $i$ & $\vdots$ & & 1 \\
\hline 10 & & $\vdots 1$ & & \\
\hline
\end{tabular}

Table 1. Truth table of an example logic function.

\begin{tabular}{cccc|c}
\hline$x_{1}$ & $x_{2}$ & $x_{3}$ & $x_{4}$ & $\mathrm{~F}$ \\
\hline 0 & 0 & 0 & 0 & \\
0 & 0 & 0 & 1 & \\
0 & 0 & 1 & 0 & \\
0 & 0 & 1 & 1 & 1 \\
0 & 1 & 0 & 0 & 1 \\
0 & 1 & 0 & 1 & 1 \\
0 & 1 & 1 & 0 & 1 \\
0 & 1 & 1 & 1 & 1 \\
1 & 0 & 0 & 0 & 1 \\
1 & 0 & 0 & 1 & \\
1 & 0 & 1 & 0 & \\
1 & 0 & 1 & 1 & 1 \\
1 & 1 & 0 & 0 & \\
1 & 1 & 0 & 1 & \\
1 & 1 & 1 & 0 & \\
1 & 1 & 1 & 1 & \\
\hline
\end{tabular}

Table 2. Karnaugh map of Table 1.

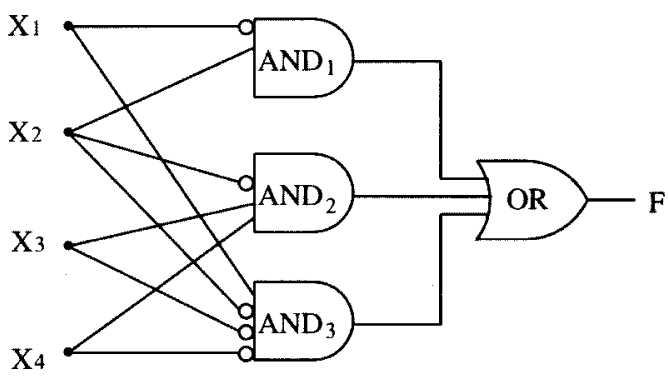

Fig. 1. AND-OR network.

\section{A Boolean Algebra Based Learnable Net- work}

We consider an n-input variables $x_{1}, x_{2}, \cdots, x_{n}$ and one output logic network. The corresponding architecture is shown in Fig.2, where a circle node has modifable parameters while the AND node and the OR node have none and node function in the same layer are of the same type, as described below :

Layer 1: Each node in this layer is a connection function and yields the direct connection or the inversed connection or the 1-constant connection or the 0 -constant connection, to corresponding AND gates. The connection function can be described by a one-input oneoutput neuron function and its node function is given by

$$
Y_{i j}=f(x)=\frac{1}{1+e^{-k\left(w_{i j} x_{i}-\theta_{i j}\right)}} \cdots \cdots \cdots \cdots
$$




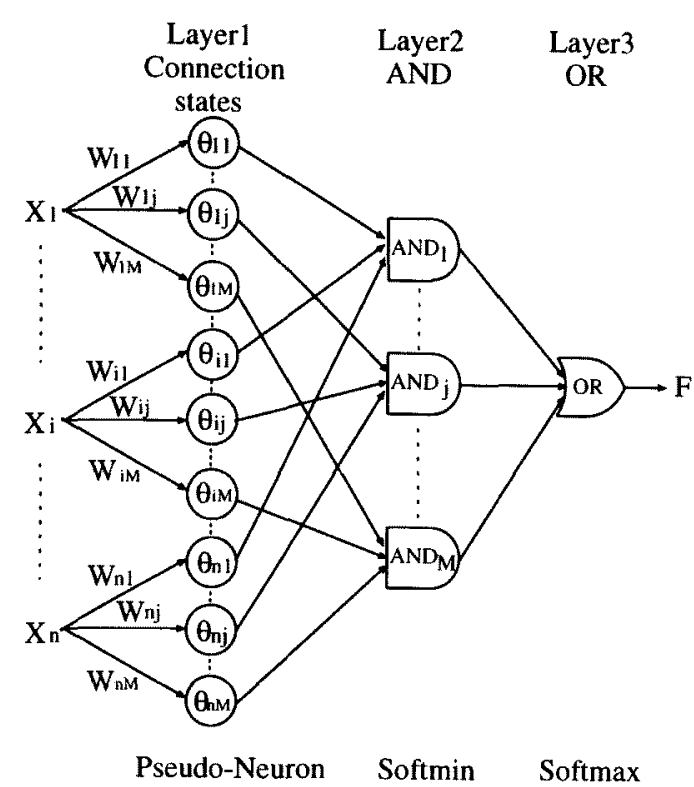

Fig. 2. A Boolean algebra based learnable network.

where $k$ is a constant proportional to the neuron gain, $w_{i j}$ and $\theta_{i j}$ are the weight from input $x_{i}$ to the $\mathrm{j}$-th neuron and the threshold of the ij-th neuron. Since the input $x_{i}$ has only two constants, 0 and 1 , for a large $k$ (e.g., $k=10$ ), there are only four cases for different weights $w_{i j}$ and threshold $\theta_{i j}$ as follows.

Case 1a: $0 \leq w_{i j}<\theta_{i j}$, for example $w_{i j}=1.0$ and $\theta_{i j}=1.5$, as shown in Fig.3(a), corresponds to the 0constant connection.

Case 1b: $w_{i j}<0<\theta_{i j}$, for example $w_{i j}=-1.0$ and $\theta_{i j}=0.5$ or 1.5 , as shown in Fig.3(b), produces the same 0 -constant connection.

Case 2: $w_{i j}<\theta_{i j}<0$, for example $w_{i j}=-1.0$ and $\theta_{i j}=-0.5$, as shown in Fig.3(c), gives the inversed connection.

Case 3: $0<\theta_{i j} \leq w_{i j}$, for example $w_{i j}=1.0$ and $\theta_{i j}=0.5$, as shown in Fig.3(d), presents the direct connection.

Case 4a: $\theta_{i j} \leq 0 \leq w_{i j}$, for example $w_{i j}=1.0$ and $\theta_{i j}=-0.5$ or -1.5 , as shown in Fig. $3(\mathrm{e})$, shows the 1-constant connection.

Case $4 b: \theta_{i j} \leq w_{i j}<0$, for example $w_{i j}=-1.0$ and $\theta_{i j}=-1.5$, shown in Fig.3(f), illustrates the 1-constant connection.

Therefore, the connection states can be described by the connection neuron. Since the connection neuron is a one-input one-output unit and its weight and threshold will not appear in practical hardware implementations which will be discussed in our next work, we call it as the pseudo-neuron. As the values of $w_{i j}$ and $\theta_{i j}$ of the pseudo-neuron change, the connection function varies accordingly, thus exhibiting various states of the connection. Further, the function of the pseudo-neuron is clearly differential. Their derivatives of the function can be expressed as,

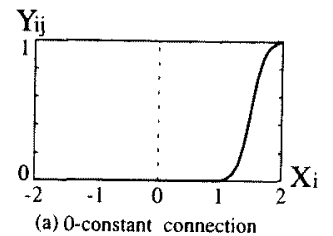

(a) 0-constant connection $0<W_{i j}<\theta_{i j}$ (e.g., $\left.W_{i j}=1.0, \theta_{i j}=1.5\right)$ $Y_{i j}$

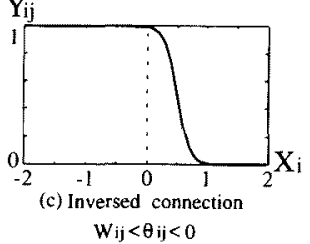

(e.g., $\left.W_{i j}=-1.0, \theta_{i j}=-0.5\right)$

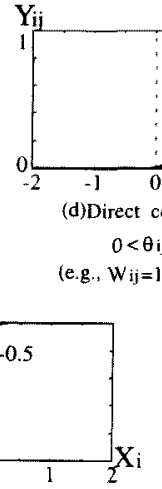
$0<\theta_{i j}<W_{i j}$

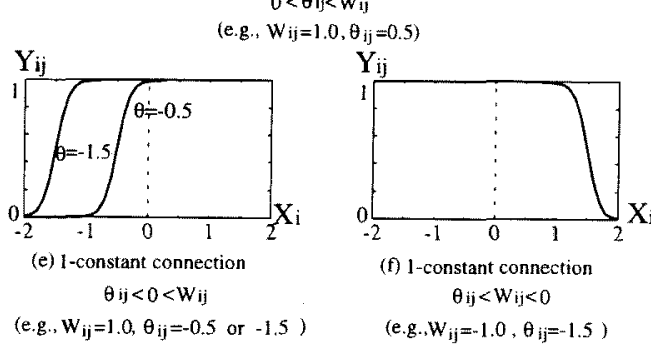

Fig. 3. Pseudo-neuron output characteristics and its equivalent connection states.

$$
\frac{\partial Y_{i j}}{\partial w_{i j}}=-k x_{i} f(x)(1-f(x))
$$

and

$$
\frac{\partial Y_{i j}}{\partial \theta_{i j}}=k f(x)(1-f(x))
$$

Layer 2: A node in layer 2 corresponds to the AND operation. The AND operation of the input variable yields the value 1 if and only if all input variables are simultaneously 1 . For any $\mathrm{n}$-variable logic function, only $2^{n-1}$ AND gates will be necessary. In order to do learning with gradient descent, derivatives of the AND function are needed. However, the derivatives of the AND function do not exist. Here, we use a softmin operator.

$$
f_{\min }=\frac{\sum x_{i} e^{-\mu x_{i}}}{\sum e^{-\mu x_{i}}}
$$

where $\mu$ is a positive constant. Fig.4 shows the characteristics of a two-variables softmin function with different parameters $\mu$. The softmin function produces a same result as the AND operation in the limit. Furthermore, the function is also clearly differential and its derivative can be written as: 


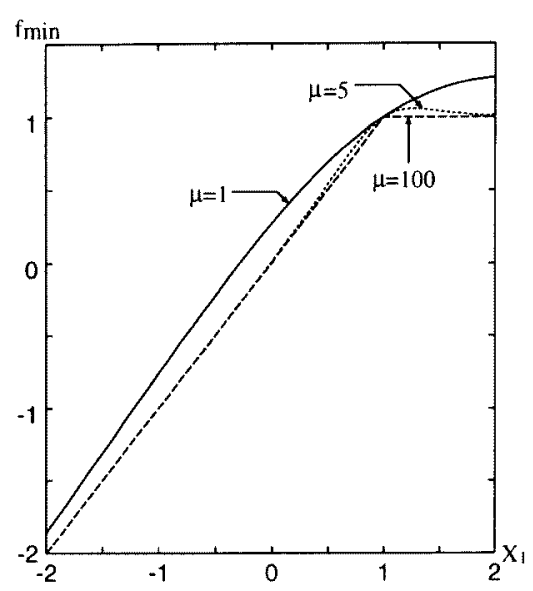

Fig. 4. The characteristics of the two-variables softmin function with $X_{2}=1$.

$$
\frac{\partial f_{\min }}{\partial x_{i}}=\frac{\sum_{l}\left(1-\mu x_{i}+\mu x_{l}\right) e^{-\mu\left(x_{i}+x_{l}\right)}}{\left(\sum_{l} e^{-\mu x_{i}}\right)^{2}} \cdots \cdots
$$

Layer 3: This node gives the OR operation of the product terms. The OR operation yield the value 1 whenever at least one of the variables is 1 , and 0 otherwise, where each $x_{1}, x_{2}, \cdots, x_{n}$ assumes the value 0 or 1. For learning purposes, we use a softmax operator:

$$
f_{\max }=\frac{\sum x_{i} e^{\nu x_{i}}}{\sum e^{\nu x_{i}}}
$$

where $\nu$ is a positive constant. Fig.5 shows the characteristics of a two-variables softmax function with different parameters $\nu$. It shows a same result as the $O R$ operator in the limit. The softmax. function is also differential and its derivative may be calculated as:

$$
\frac{\partial f_{\max }}{\partial x_{i}}=\frac{\sum_{l}\left(1+\nu x_{i}-\nu x_{l}\right) e^{\nu\left(x_{i}+x_{l}\right)}}{\left(\sum_{l} e^{\nu x_{l}}\right)^{2}}
$$

The learnable network described above is a multi-layer feed-forward network in which each node performs a particular function (node function) on incoming signals using a set of parameters specific to this node. The form of node function varies from layer to layer, and each node function can be defined by prior knowledge on the network. Furthermore, modifiable parameters are present only on layer 1 . The other weights are fixed at unit.

\section{Learning Mechanism}

The learnable network mentioned above is a feedforward network with continuous functions. Thus, the error back-propagation algorithm will be valid for the network. A learning rule for the network can be readily derived from the condition of least squared error between the actual output $F_{l}$ and the desired output $T_{l}$ defined as

$$
E=\frac{1}{2} \sum_{l}\left(T_{l}-F_{l}\right)^{2}
$$

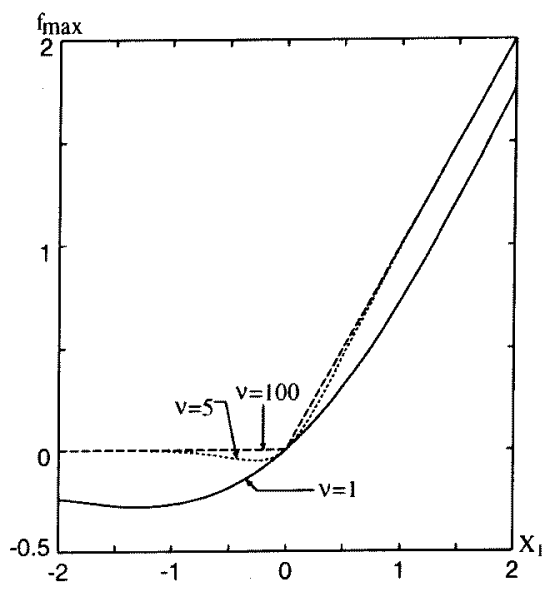

Fig. 5. The characteristics of the two-variables softmax function with $X_{2}=0$.

Since the minimization of the error requires the weight and threshold changes to be in the negative gradient direction, we take

$$
\Delta W=-\eta \nabla E
$$

and

$$
\Delta \theta=-\eta \nabla E
$$

where $\eta$ is a positive constant called the learning constant. For the weights $w_{i j}$ and the thresholds $\theta_{i j}$, we must differentiate with respect to the $w_{i j}$ and $\theta_{i j}$ which are deeply embedded in Eq.(10). Using the chain rule, we obtain

$$
\begin{aligned}
& \Delta w_{i j}=-\eta \frac{\partial E}{\partial w_{i j}} \\
& =-\eta \sum_{l} \frac{\partial E}{\partial F_{l}} \cdot \frac{\partial F_{l}}{\partial w_{i j}} \\
& =\eta \sum_{l}\left(T_{l}-F_{l}\right) \cdot \frac{\partial F_{l}}{\partial A N D_{j}} \cdot \frac{\partial A N D_{j}}{\partial w_{i j}} \\
& =\eta \sum_{l}\left(T_{l}-F_{l}\right) \cdot \frac{\partial F_{l}}{\partial A N D_{j}} \cdot \frac{\partial A N D_{j}}{\partial Y_{i j}} \cdot \frac{\partial Y_{i j}}{\partial w_{i j}}
\end{aligned}
$$

and

$$
\begin{aligned}
\Delta \theta_{i j} & =-\eta \frac{\partial E}{\partial \theta_{i j}} \\
& =\eta \sum_{l}\left(T_{l}-F_{l}\right) \cdot \frac{\partial F_{l}}{\partial A N D_{j}} \cdot \frac{\partial A N D_{j}}{\partial Y_{i j}} \cdot \frac{\partial Y_{i j}}{\partial \theta_{i j}}
\end{aligned}
$$

which is the learnable rule for the network. Using the above learning rules Eqs.(13),(14), the derivatives Eqs.(4),(5), and Eqs.(7),(9) derived above, where $F_{l}$ is $f \max$, the output of the OR, AND $j$ is $f \min _{j}$, the output of $\mathrm{AND}_{j}$ to $f \max$, and $Y_{i j}$ is the output of connection function to $f \min$, respectively, we can carry out 
the back-propagation to the networks. It may be noted that the learnable rules of the network are quite similar to traditional back-propagation algorithm of neural networks. The essential difference between them is that the gradient descent procedure effectively works on only one layer, rather than all layers. Here, we have extended the logic network concept by replacing the connection states, logic AND operator and logic OR operator with a pseudo-neuron, softmin and softmax characterized by their own continuous functions, respectively. Another significant difference between the learnable networks and neural networks is that the learnable networks use the logic AND and logic OR operation and by slight modifications of the softmin and softmax functions, the learning rule would lead to a simple logic signal. Thus, by digitizing the connection states and their changing into a discrete one, the networks and their learning can be implemented by simple logic circuits, which is available for hardware implementations. The details will be discussed in our next works.

\section{Simulation Results and Discussions}

In order to demonstrate the capabilities of the algorithm for learning networks, we used several benchmark data sets. The first set is the exclusive-or problem. The problem has become standard in neural network literature, because it cannot be solved by a simple linear classifier. We used a two-AND-gate and one-OR network and initialized the weights and thresholds randomly, as shown in Fig.6(a). The network was trained by the algorithm with all parameters equal to 1.0. All of the networks were trained with updating after each train-

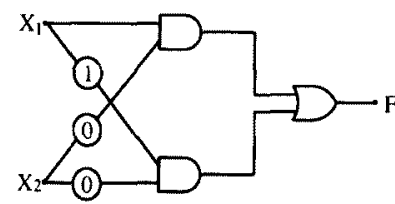

( a )

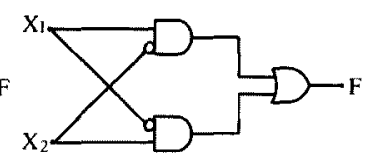

(b)
Fig. 6. The AND-OR network for the exclusive OR problem : (a) befoe and (b)after training.

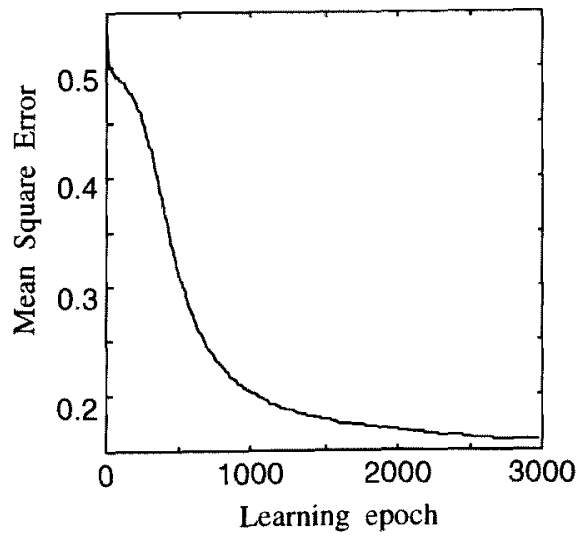

Fig. 7. Sum-squared error during training. ing data. The sum-squared error on the training set during training is plotted in Fig.7. In Fig.7, we see that the learning network lead to minimization of the error function when the exclusive-or data are employed. The final network after training is shown in Fig.6(b).

The algorithm under consideration has also been tested with different parameters. During learning, the parameters $\eta, k, \mu$ and $\nu$ in Eqs.(11), (12), (3), (6) and Eq.(8) were chosen as $\eta=1.0, k=1.0, \mu=1.0$ and $\nu=1.0$. Fig. 8 is drawn to illustrate the effect of varying the learning constant $\eta$. It was observed that $\eta=10$ gives the best results. A larger $\eta$ is seen to provide better performance when $\eta>10$. The results of using a larger $\eta$ than 10 are shown in Fig.9, demonstrating failure to achieve global convergence, perhaps because of overshooting.

In Fig. 10 we observe the effect of the sharpness parameter $k$ of the sigmoid pseudo-neuron. A large $k$ leads to notably better learning performance. But, an over large $k(\mathrm{e} . \mathrm{g}, k \geq 5)$ produces local minimum and results in a comparatively poor performance, performance, perhaps also owing to the overshooting during learning.

Fig.11 demonstrates the effects of the softmin parameter $\mu$ and the softmax parameter $\nu$ on the learning performance. For each of the two parameters ( (a) and

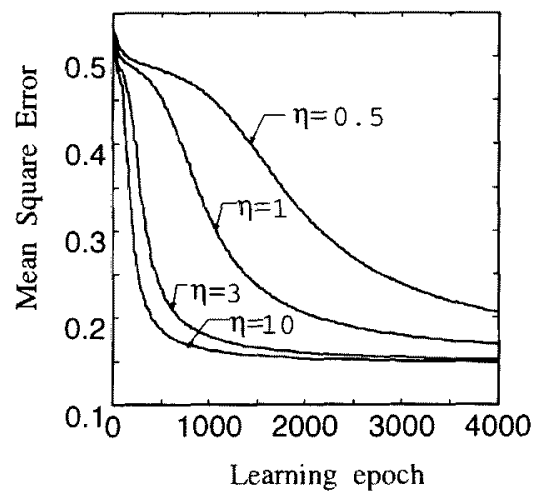

Fig. 8. Learning performance with different $\eta$.

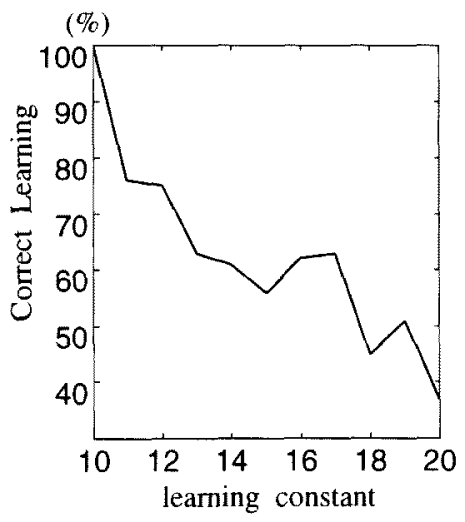

Fig. 9. Correct lerning (\%) versus different $\eta$. 


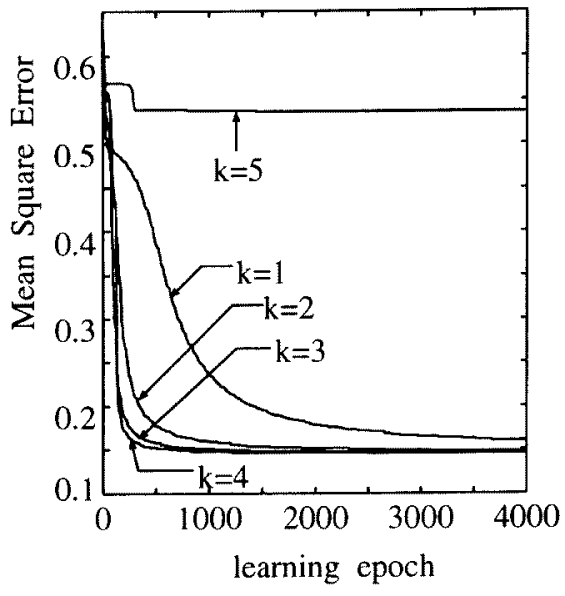

Fig. 10. Learning performance with different $k$.

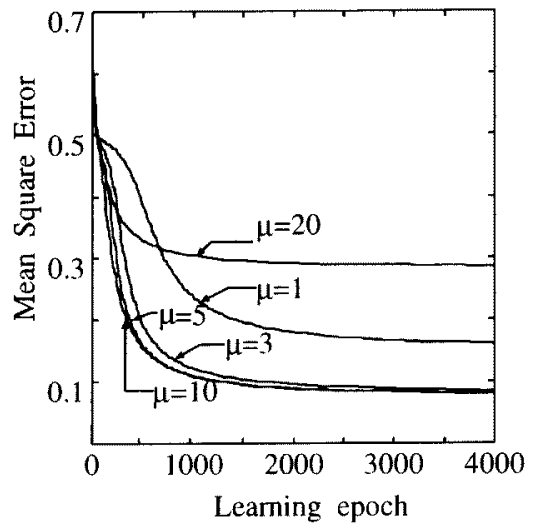

(a)

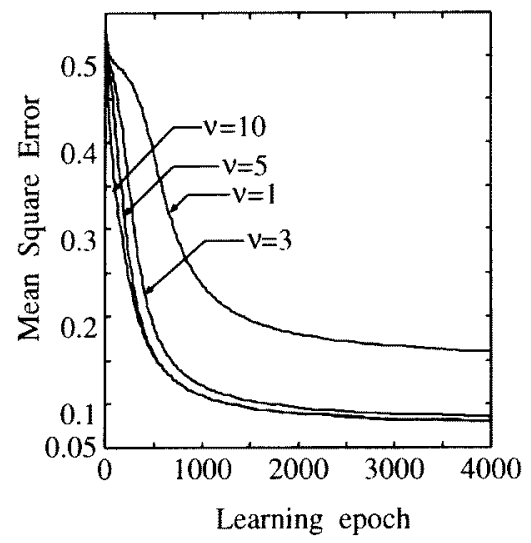

(b)

Fig. 11. Learning performance with different $\mu$ (a) and $\nu(\mathrm{b})$.

(b) of Fig.11), a larger parameter learns to achieve the global minimum faster, and its performance is excellent. Unfortunately, it is also not so good for an over large parameter, e.g., $\mu, \nu \geq 10$.

Furthermore, from Fig.6, we can see that the 1-

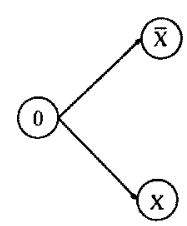

(a)

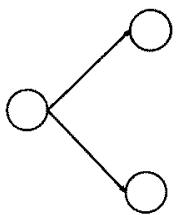

(b)
Fig. 12. The cell division in learning network (a) and cytology(b).

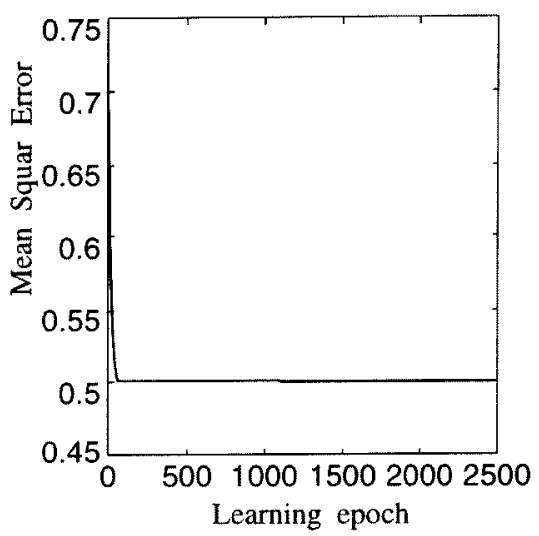

(a)

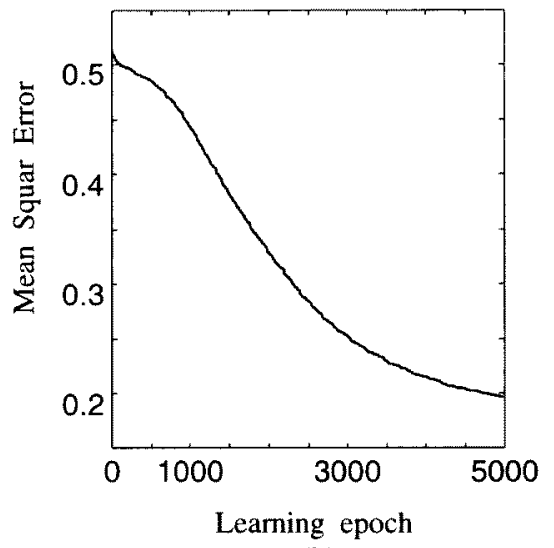

(b)

Fig. 13. Learning performance for different numbers of AND cells. (a) 1-AND and (b) 5-AND cells.

constant connection pseudo-neuron has been changed to the inversed connection, the 0 -constant connection pseudo-neuron has been divided into the direct connection and the inversed connection (Fig.12), corresponding to the cell division in cytology. We have also tested the algorithm for different numbers of AND cells (such as 1 , and 5) to the exclusive OR problem. Fig.13 shows the simulation results. A single AND cell is obviously incapable of realizing the exclusive OR problem. The 5-AND cells lead to a global minimum, as expected. Furthermore, examining the network after learning (Fig.14(a)), we can see the AND1, AND3, and $\mathrm{AND}_{4}$ cells the AND2 and AND5 cells have the same inputs. Thus, the redundant ones can be reduced by basic 


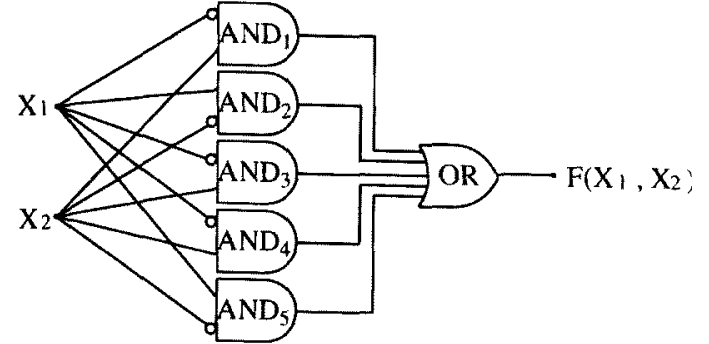

(a)

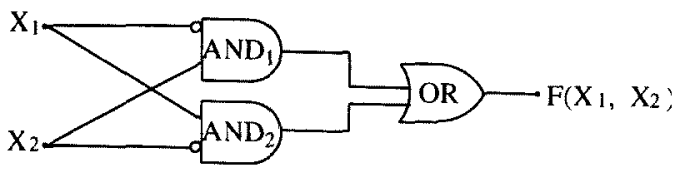

(b)

Fig. 14. Five AND cells network for the exclusive OR problem : (a)after learning and (b) after reduction.

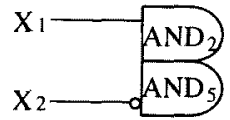

(a)

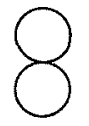

(b)
Fig. 15. The cell adhesion in learning network (a) and cytology (b).

Table 3. Truth table of a center symmetry detecting problem.

\begin{tabular}{|c|c|c|c|c|}
\hline$x_{3} X_{2}$ & 00 & 01 & 11 & 10 \\
\hline 00 & 1 & & & \\
\hline 01 & & & & 1 \\
\hline 11 & & & 1 & \\
\hline 10 & & 1 & & \\
\hline
\end{tabular}

Boolean algebra. Thus, we have realized a reduction of five AND cells to two AND cells, shown in Fig.14(b). The reduction can be viewed as the cell adhesion in cytology, shown as Fig.15.

In the next example we illustrate a center symmetry detecting problem as shown in Table 3 . For this problem, we selected eight AND cells. The connection states, e.g., the weights and the thresholds to the pseudo-neurons were initialized randomly. The parameters of $\eta, k, \mu$ and $\nu$ were set to $3.0,1.0,2.0$ and 5.0 , respectively. Simulation had an order from 0000 to 1111 for the 16 training set exemplars. Fig.16 provides the learning performance for the learnable binary network. As can be seen, the network has learned the problem correctly. The corresponding network after the learning is shown in Fig.17(a). However, clearly, the AND1, AND3, AND4, and AND7, can be eliminated, as shown in Fig.17(b). It can be viewed as, the death of the cells in cytology. There are several types of the death of cells,

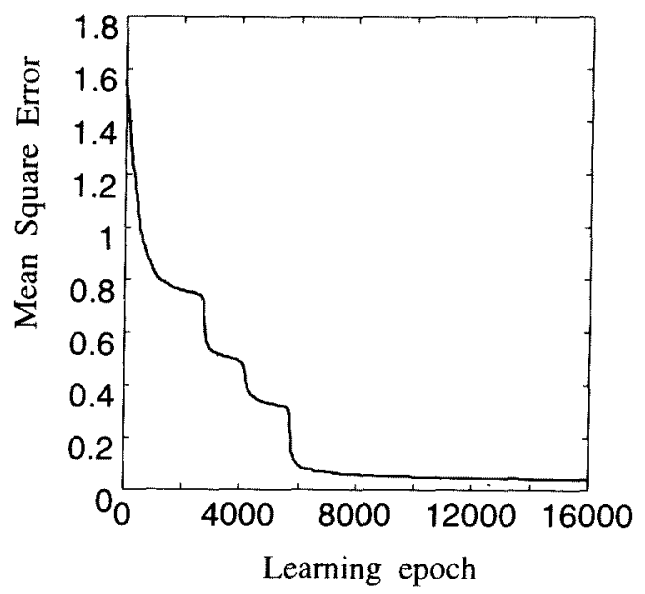

Fig. 16. Learning performance to the Table 3.

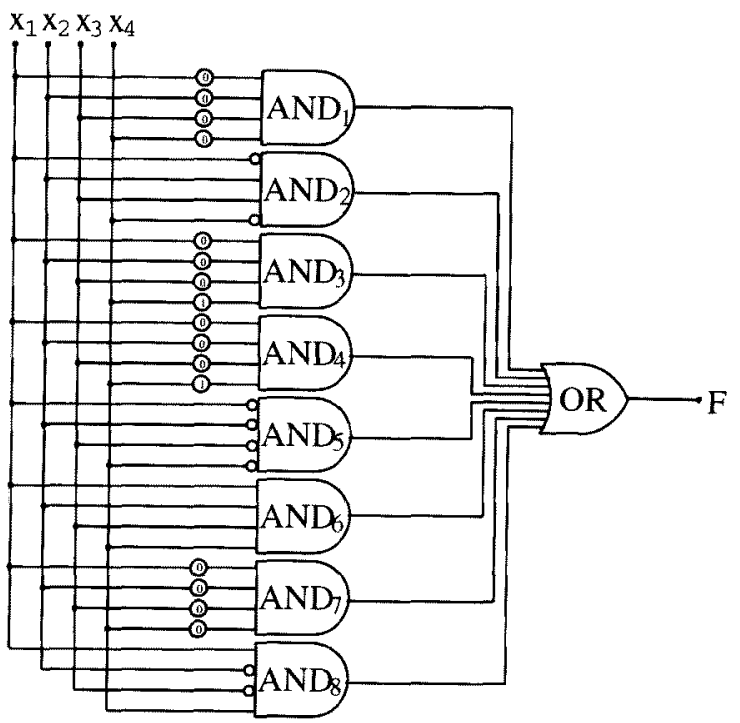

(a)

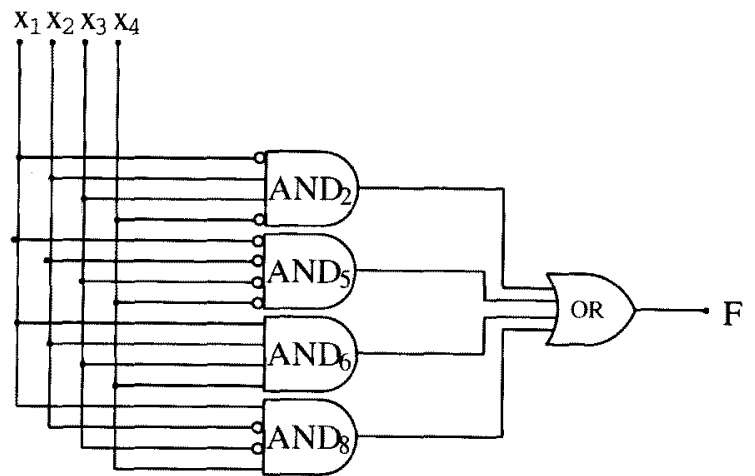

(b)

Fig. 17. The network for Table 3: (a) before and (b) after reduction. 


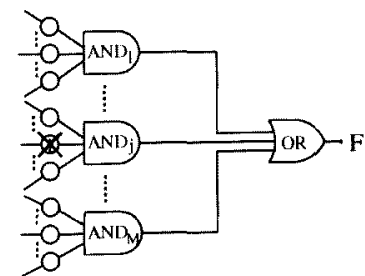

(a)

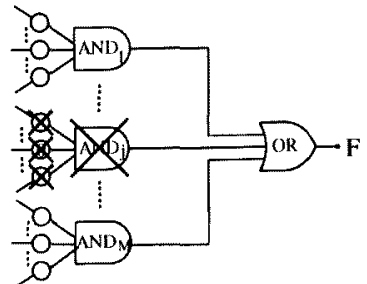

(b)

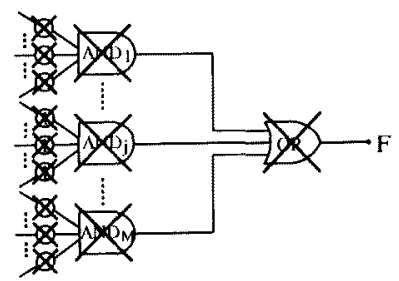

(c)

Fig. 18. Correspondence of the death of a single cell (a), and cluster cells ((b) and (c)).

Table 4. Truth table.

\begin{tabular}{|c|c|c|c|c|}
\hline$x x_{2}$ & 00 & 01 & 11 & 10 \\
\hline 00 & & 1 & & \\
\hline 01 & 1 & 1 & & \\
\hline 11 & & 1 & & \\
\hline 10 & & 1 & & \\
\hline
\end{tabular}

for example, the death of single cell, and the death of cluster cells. If a cell is a 1-constant pseudo-neuron to an AND cell, the cell can be deleted, corresponding to the death of the single cell (Fig.18(a)). If one or more cells is a 0-constant pseudo-neuron to an AND cell, the AND cell and all cells to the AND cells can be relieved, corresponding to the death of a small cluster cell (Fig.18(b)). Further, if all cells to an AND cell are 1-constant pseudo-neurons, the OR cell, all AND cells to the OR cell and all cells to these AND cells will be removed, corresponding to the death of a large cluster cell (Fig.18(c)). Finally, we give an example to show how the learnable network works on a problem on which the reduction can be performed. Supposed we have a 2 -variable training set shown in Table 4 . We used a network of Fig.19(a) initialized randomly and learning is performed on the network. Fig. 20 shows the learning performance of the network. After being trained by $\mathrm{Ta}$ ble 4, the network of Fig. 19(a) has been reduced to the network as shown in Fig.19(b) in which only $\mathrm{AND}_{3}$ and $\mathrm{AND}_{7}$ are required for the function and others can be eliminated. It is obvions that the learning network provides a good learning characteristics and yields a minimal realization as the Kanaugh map method. However it should be noted that the learning is not guranteed to work always so well because it depends strongly on initial parameters.

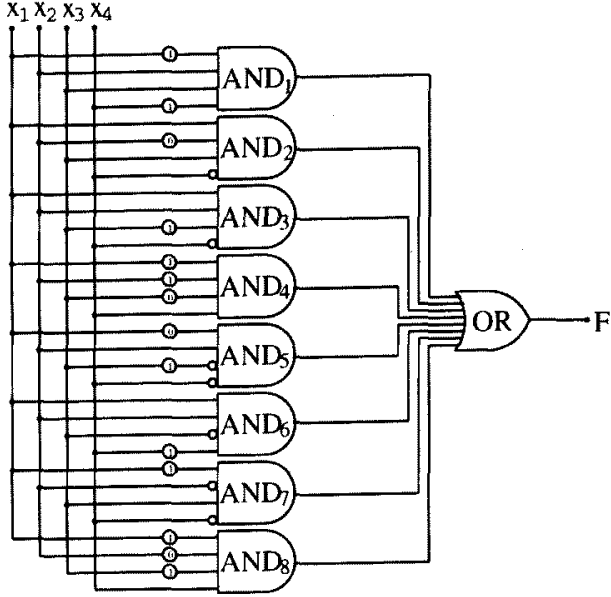

(a)

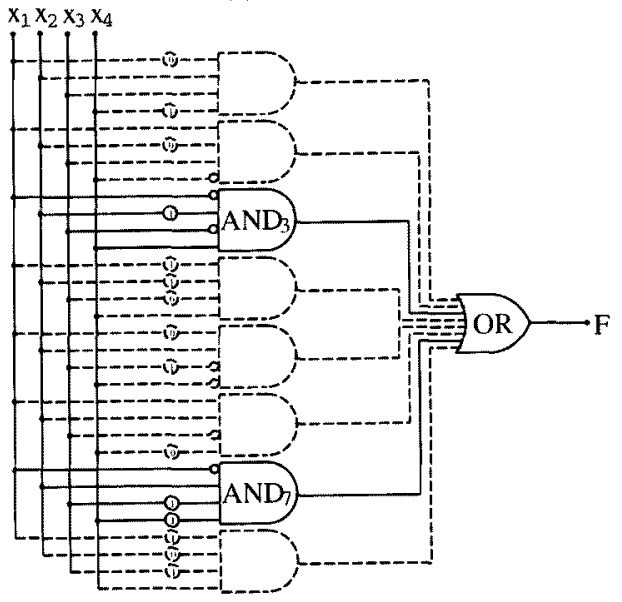

(b)

Fig. 19. The network for Table 4: (a) before and (b) after learning.

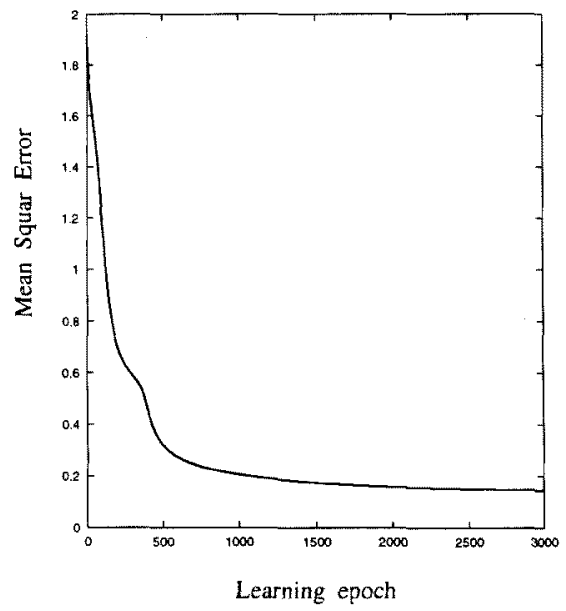

Fig. 20. Learning performance to the truth table 4. 


\section{Conclusions}

The learnable network presented in this paper introduces a pseudo-neuron, a softmin function and a softmax function to represent the connection states, the logical AND operator and the logical OR operator of logic network, and uses a gradient descent technique to train the logical AND-OR network with these continuous functions. Thus, the logical AND-OR based networks are capable of learning just like the traditional neural networks. Importantly, the learnable networks does not involve any additional analog or digital realization of neuron functions, which is available for highspeed high-density hardware implementations. Furthermore, for digital system, the learning network can be simplified to logical AND-OR network. The learning algorithm, making use of traditional back-propagation algorithms, has been described, and its effectiveness illustrated.

\section{Acknowledgement}

This work was supported in part by the International Communications Foundation.

(Manuscript received November 16, 1998, revised April 26, 1999)

\section{References}

(1) D.E. Rumelhart, G.E. Hinton and R.J. Williams: "Learning internal representations by error propagation," in Par allel Distributed Processing, Vol.1, D.E. Rumelhart and J.L. McClelland, Eds. Cambridge, MA : MIT Press, 1986, pp. 318362.

(2) D.Z. Anderson, Ed, Neural Information Processing Systems, AmericanInstitute of physics Conference Proceedings, Denver, Nov. 1987

(3) J.R. Anderson: Neurocomputing. Cambridege, MA : MIT Press, 1988.

(4) J.S. Denker: Neural Networks for computing, American institute of physics conference Proceedings, Vol.151, Snowbird UT, Apr. 1986

(5) S.Y. Kung, and J.N. Hwang: "Parallel architectures for artificial neural nets," IEEE ICNN Conference Proc., 1988, pp. 165-172.

(6) D.B. Schwartz, and R.E. Howard: "A programmable analog neural network chip." IEEE cust. Intg. Ccts. Conf., 1988, pp. $12 \cdot 2 \cdot 1-12 \cdot 2 \cdot 4$.

(7) M.A. Silvotti, M.R. Emerling, and C.A. Mead: "VLSI architectures for implementation of neural networks," Am. Inst. Phys., pp. 408-413, 1986

(8) - Z. Tang, O. Ishizuka and H. Matsumoto: "AN asymmetvic interconnection neural network and its applications," Proc. of International Conference on Fuzzy \&6 Neural Networks, 1990 , pp. 645-649.

(9) D.W. Tank and J.J. Hopfield: "Simple 'neural' optimization network : an A/D converter, signal decision circuit, and a liner programming circuit," IEEE Trans. on Circuits and Systems, Vol.CAS-33, 1986, pp. 553-541.

(10) Z. Tang, O. Ishizuka, M.Kuratsu and K. Tanno: "Binary learning machine using back-propagation technique"-invited paper, Proc. IEEE International Conference on Systems, Man and Cybernetics, SanDiego, USA, TP7.6, Oct., 1998
Zheng Tang (Non-member) He was born in 1959.He re-

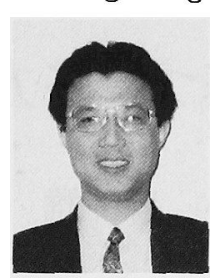
ceived the B.S. degree from Zhejiang University, Zhejiang, China in 1982 and the M.S. degree and the Ph.D. degree from Tsinghua University, Beijing, China in 1984 and 1988 respectively. From 1988 to 1989 , he was Instructor in Institute of Microelectronics at Ts inghua University. In 1989, he joined Miyazak University, Miyazaki, Japan, where he is currently Associate Professor in Department of Electrical \& Electronic Engineering. His current research interests include neural networks, multiple-valued logic, fuzzy control and analog integrated circuit design.

Makot Kuratsu (Non-member) He was born in 1974. He

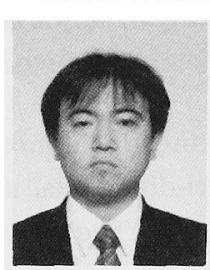
received the B.S. and M.S.degree in electrical and electronic engineering from Miyazaki University in 1997 and 1999 , respectively. $\mathrm{He}$ is currently with Kyusyu Ando Electric Corpo ration. His research interests are neural networks.

Okihiko Ishizuka (Non-member) He was born in 1939

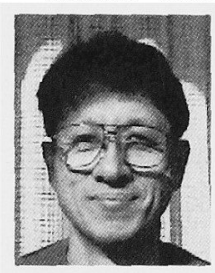
He received the B.E. degree in 1964 from Kagoshima University, and the M.E. degree in 1966 and the D.E. degree in 1978 from Kyusyu University. He is a Professor of Electrical \& Electronic Engineering, Miyazaki University, Miyazaki, Japan. His research interests are network synthesis, and circuit analysis on multi-valued logic, threshold logic and fuzzy logic: Dr.Ishizuka is a member of IEEE and Executive Subcommittee of Computer Society Technical Committee on Multi-Valued Logic.

Koichi Tanno (Non-member) He was born in 1967. He re-

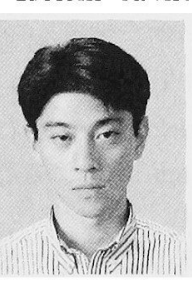
ceived the B.S. and M.S. degrees in Faculty of Engeering from Miyazaki University, in 1990 and 1992, respectively. From 1992 to 1993 he joined the Microelectronics Products Development Laboraroty, Hitachi, Ltd., Yokohama Japan. He has been engaged in research in the low supply voltage continuous-time filter. Since 1994, he has been with the Faculty of Engineering, Miyazaki University where he is now a Research Associate in the Department of Electrical \& Electronic Engineering. His main research interests are bipolar and CMOS analog circuits and hardware realization of fuzzy controller and neural networks. Mr.Tanno is a member of IEEE. 